\title{
Measurement of pre-self-balanced surface residual stresses induced by milling in titanium alloys and the FEM validation
}

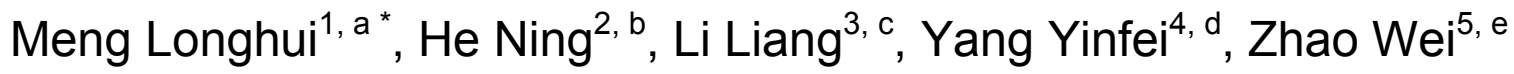 \\ ${ }^{1,2,3,4,5}$ College of Mechanical and Electrical Engineering, Nanjing University of Aeronautics and \\ Astronautics, Nanjing, Jiangsu, 210016, China \\ aemail: menglonghui@nuaa.edu.cn, bemail:drnhe@nuaa.edu.cn, email:liliang@nuaa.edu.cn, \\ demail:yyfgoat@nuaa.edu.cn, eemail:nuaazw@nuaa.edu.cn
}

Key Words : residual stress, corrosion, bending deflection, Finite Element Method (FEM)

\begin{abstract}
Flank milling was used to machine the titanium alloy workpiece, and high residual stresses were induced by milling in the superficial layers. The stress layers were corroded gradually and the changes of workpiece's bending deflection were measured. The stress values in the upper layers were calculated out based on the values in the bottom layers and the changes of the bending deflection. At last, the stress values got by this method are prior to the self-balanced state, so the result rules out the influence from the size and shape of the workpiece. The deformations of workpieces with different sizes and shapes can be predicted accurately based on these stress values. The correctness of this method has been verified by Finite Element Method (FEM).
\end{abstract}

\section{Introduction}

Metal cutting is a severe plastic deformation process with high temperature, high pressure, high strain rate, and is also affected by thermal stress and phase transformation ${ }^{[1]}$, and there will be residual stresses induced by machining in the surface layers. Usually the distribution of the stresses is very shallow (no more than $0.2 \mathrm{~mm}$ ), but the changing rate is very high along the depth direction.

Titanium and its alloys are widely used in aerospace field because of their light density, high strength, good resistance to corrosion and high temperature ${ }^{[2]}$, but the residual stresses will seriously affect the dimensional accuracy and service performance of the workpieces ${ }^{[3]}$.

Since the 1930's, more than ten methods have been used to measure the residual stresses, and they can mainly be divided into destructive technique and nondestructive technique. The destructive technique mainly includes hole drilling-method, ring-core method, deep-hole method, sectioning technique, contour method, etc., the most used one is hole-drilling method; the nondestructive technique mainly includes barkhausen noise method, X-ray diffraction (XRD) method, neutron diffraction method, ultrasonic method, etc., the most used one is X-ray diffraction method ${ }^{[4-9]}$.

Nowadays the most used method for measuring surface residual stresses is XRD method combined with layer removal. The formation of the residual stresses' final state will go through two steps: 1 , the formation of the surface residual stresses induced by many factors during the process of machining; 2, the residual stresses will be redistributed to get a self-balanced state. The size and shape of the workpiece will seriously affect the self-balanced result. So, in spite of the same cutting tool, the same material of the workpieces, the same cutting parameters(cutting speed, feed rate and cutting thickness) and the same cutting conditions(with cutting fluid or not), the final surface residual stresses may be different. As to predict the deformations of the workpieces accurately, it is 
necessary to measure the stresses values prior to the self-balanced state. The stress values got by XRD method cannot predict the deformations accurately as they are already in the self-balanced state $^{[10]}$.

This paper presents a new measuring method, which is based on the changes of the workpiece's bending deflection, calculate the stress values from the bottom layers to the upper layers. Finally, the stress values got by this method are prior to the self-balanced state. The correctness of this method will be verified by finite element method (FEM) in this paper.

\section{Calculation Principle}

Fig. 1 represents a schematic drawing of the workpiece, the length is $L$, and the thickness is $h$. The neutral layer is always in the middle position of the workpiece, when any stress layer has been removed, the neutral layer will be changed as shown in Fig.1.

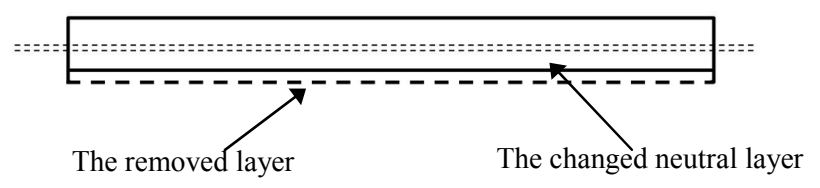

Fig.1 The sketch map of the corroded layer and the changed position of the neutral layer

The bending deflection of the workpiece will also be changed by removing any stress layer, as shown in Fig.2.

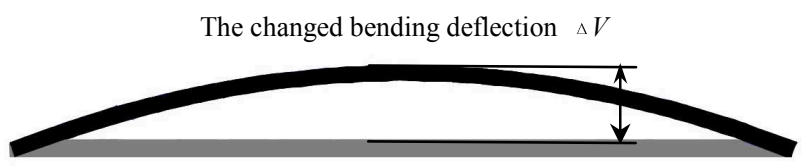

Fig.2 The sketch map of the change of the workpiece's bending deflection

When the bending deflection is tending towards stability, $N$ layers have been removed, the thickness of the last layer is $\Delta h_{N}$, and the thickness of the remaining part is $h_{N}$. When the last layer has been removed, the change of the bending deflection can be seen as completely caused by releasing the residual stress $\sigma_{N}$ in the $N$ th layer, so the bending moment can be described as: $M_{N}=F_{N} l_{N}=\sigma_{N} \cdot b \cdot \Delta h_{N} \cdot\left(h_{N}+\Delta h_{N}\right) / 2$.

$F_{N}$ is the residual force in the Nth layer, $l_{N}$ is the distance between the Nth layer and the neutral layer before removing the $N$ th layer, $b$ is the width of the workpiece. When the $N$ th layer has been removed, the change of the bending moment can be described as: $\Delta M_{N}^{N}=-M_{N}=-\sigma_{N} \cdot b \cdot \Delta h_{N} \cdot\left(h_{N}+\Delta h_{N}\right) / 2$.

The subscript $N$ means the change of the moment caused by the residual stress in the $N$ th layer, the superscript $N$ means it is the $N$ th time to remove stress layer. According to the relationship between the bending deflection and the bending moment as the formula: $V_{\max }={ }^{M_{e q}} L^{2} / 8 E I$, so $\Delta M_{N}^{N}$ can also be described as: $\Delta M_{N}^{N}=8 E I_{N} \Delta V_{N} / L^{2} . E$ is the elasticity modulus of the material, 
$I_{N}$ is the second moment of inertia after removing the $N$ th layer, and its value is $I_{N}=b h_{N}^{3} / 12$. Combining the two expressions, the residual stress in the Nth layer can be described as:

$$
\sigma_{N}=-4 E h_{N}^{3} \Delta V_{N} / 3 L^{2} \Delta h_{N}\left(h_{N}+\Delta h_{N}\right)
$$

The value got by the formula above is the actual value of the residual stress in the last layer, it doesn't need any correction or compensation. The stress value in the penultimate layer can be calculated out based on the stress value in the last layer and the change of the bending deflection. The thickness of the penultimate layer is $\Delta h_{N-1}$, the change of the bending moment caused by removing the penultimate layer is: $\Delta M_{N-1}^{N-1}=-\sigma_{N} b h_{N} \Delta h_{N-1} / 2$. The removal of the penultimate layer will cause the neutral layer to move $\Delta h_{N-1} / 2$ away from the removed layer, and the change of the bending moment caused by the neutral layer's movement is: $\Delta M_{N}^{N-1}=\sigma_{N} b \Delta h_{N} \Delta h_{N-1} / 2$.

So when the penultimate layer has been removed, the change of the bending deflection is not only caused by the releasing of the residual stress in the penultimate layer, but also the residual stress in the last layer and the changed neutral layer. To change the bending deflection for $\Delta V_{N-1}$, the total bending moment needed here is: $M\left(\Delta V_{N-1}\right)=8 E I_{N-1} \Delta V_{N-1} / L^{2}$.

And the the change of the bending deflection caused just by the releasing the residual sress in the penultimate layer can be described as: $\Delta M_{N-1}^{N-1}=8 E I_{N-1} \Delta V_{N-1} / L^{2}-\sigma_{N} b \Delta h_{N-1} \Delta h_{N} / 2$.

So the stress value in the penultimate layer can be described as:

$$
\sigma_{N-1}=2 \Delta M_{N-1}^{N-1} / b \Delta h_{N-1}\left(h_{N-1}+\Delta h_{N-1}\right)
$$

Using the same way of thinking, the stress value in the nth layer from the bottom can be described as:

$$
\sigma_{N-n+1}=2 \Delta M_{N-n+1}^{N-n+1} / b \Delta h_{N-n+1}\left(h_{N-n+1}+\Delta h_{N-n+1}\right)
$$

The value of $\Delta M_{N-n+1}^{N-n+1}$ is: $\Delta M_{N-n+1}^{N-n+1}=8 E I_{N-n+1} \Delta V_{N-n+1} / L^{2} \sum_{m=N-n+1}^{N} \sigma_{m} b \Delta h_{N-n+1} \Delta h_{m} / 2$

With the calculation method above, stress values in all layers will be calculated out at last.

\section{Experimentation}

\subsection{Machining the Workpieces}

The titanium alloy work piece is as shown in Fig.3 (a), the material is Ti6Al4V. First, annealing treatment was performed on the workpiece to remove the initial residual stresses, and then flank milling was used to machine the workpiece. The parameters for the last cutting are: $v_{c}=35 \mathrm{~m} / \mathrm{min}, f_{z}=0.04 \mathrm{~mm} / \mathrm{z}, a_{e}=1 \mathrm{~mm}, a_{p}=20 \mathrm{~mm}$. The machined workpiece is as shown in Fig. 3 (b).

The thickness of the remaining part is $2.2 \mathrm{~mm}$, the length of the clamping part is $25 \mathrm{~mm}$. When measuring the bending deflection, the part chosen is $110 \mathrm{~mm}$ in the middle part of the workpiece. 


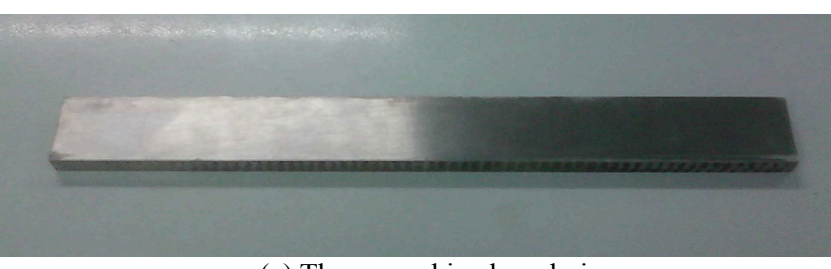

(a) The unmachined workpiece

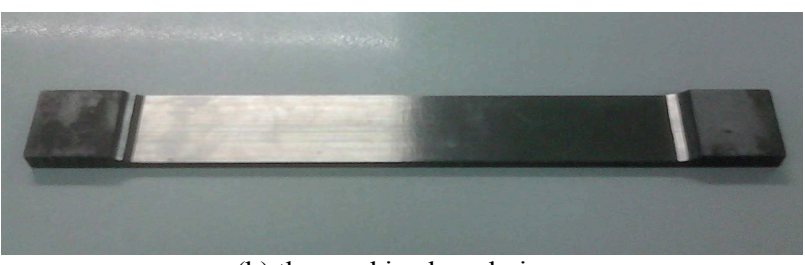

(b) the machined workpiece

Fig.3 The workpiece before and after machining

\subsection{Chemical Milling}

Here hydrofluoric acid was chosen as the corrosive as it can accelerated corrosion, and nitric acid was chosen as oxidant as it can inhibit the production of hydrogen to improve the property of the milled surface ${ }^{[11]} .704$ silicone was used to protect the surfaces that don't need to be corroded. The workpiece was dipped in the corrosive, and then the corrosive was washed away immediately.

\subsection{Measuring the Thicknesses of the Removed Layers and the Changes of the Bending Deflection}

Before chemical milling, the initial bending deflection of the workpiece was measured with laser displacement sensor, as shown in Fig.4, and a curve $S_{0}$ was got. After chemical milling for the first time, the thickness of the removed layer is $\Delta h_{1}=10.9 \mu \mathrm{m}$. The bending deflection of the workpiece was measured again and another curve $S_{1}$ was got. Subtracting $S_{0}$ from $S_{1}$, a parabola was got as shown in Fig. 8, it is the change of the workpiece's bending deflection.

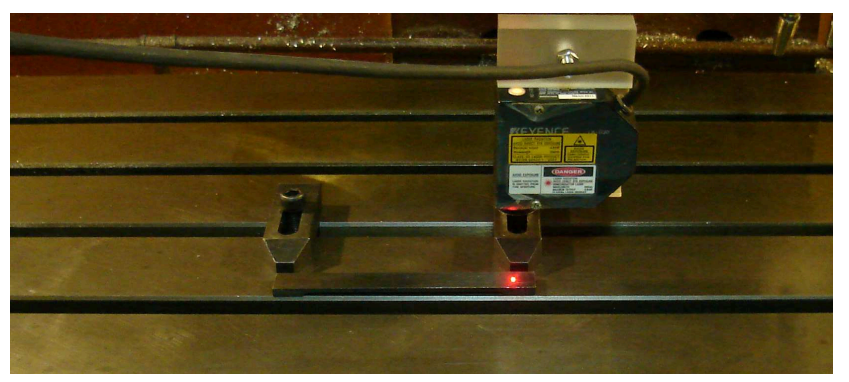

Fig.4 Measure the workpiece's bending deflection with laser displacement sensor

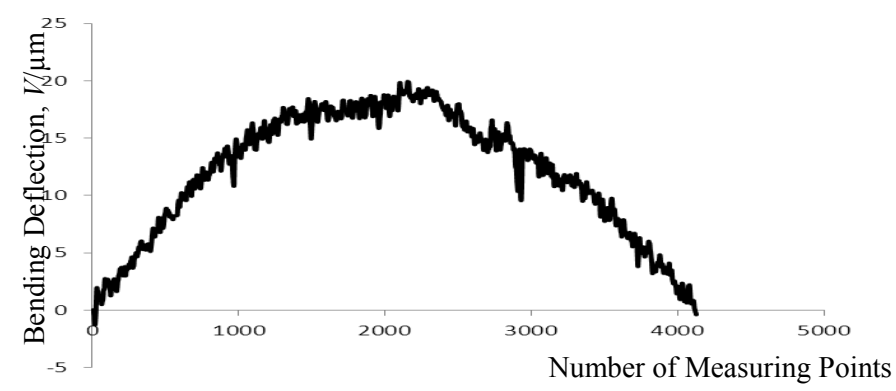

Fig.5 The change of the workpiece's bending deflection caused by the first corrosion

The steps above were repeated until there were almost no changes in bending deflection after corroding for several times, it showed that the stress layers have been removed completely. 


\section{Calculation Result}

In the experiments, when the workpiece was corroded for the first 12 times, there were obvious changes in bending deflection, but after that, there were almost no changes, so it can be concluded that the stress layers had been completely removed till then. Based on the experimental datas and the deduced calculating formulas, the stress values were got as shown in Fig.6.

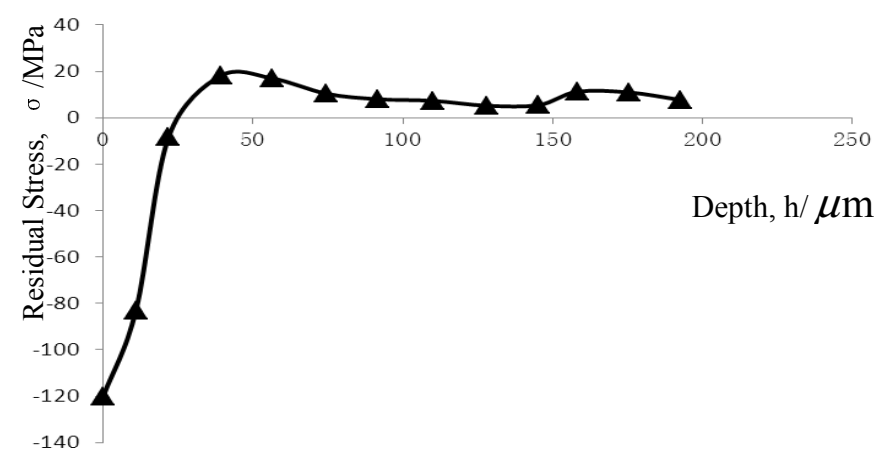

Fig.6 The curve line of calculated residual stresses

\section{FEM Validation}

A model of the workpiece was created in the software of Abaqus, and it was splited corresponding to the removed layers. The upper left and upper right edges' translational degree of freedoms were limited except for the length direction, to make sure the model can bend freely. The stress values above were applied to the model, as shown in Fig.7 (a). Since a self-balanced state was obtained, as shown in Fig.7 (b), there are some differences in the distribution of the residual stresses. As mentioned above, the differences are related to the shapes and sizes of the workpieces, and the stress values obtained from this method are prior to the self-balanced state.

The elements were removed layer by layer to simulate the process of layer removal. Each time when one layer of elements were removed, the balanced state will be disturbed and a new balanced state will be obtained. After that, the bending deflection of the workpiece will also be changed. Comparing the changes of the bending deflection calculated by the FEM to that obtained from the experiment, refer to Fig.13, it can be found that the two curves are coincident with each other very well.

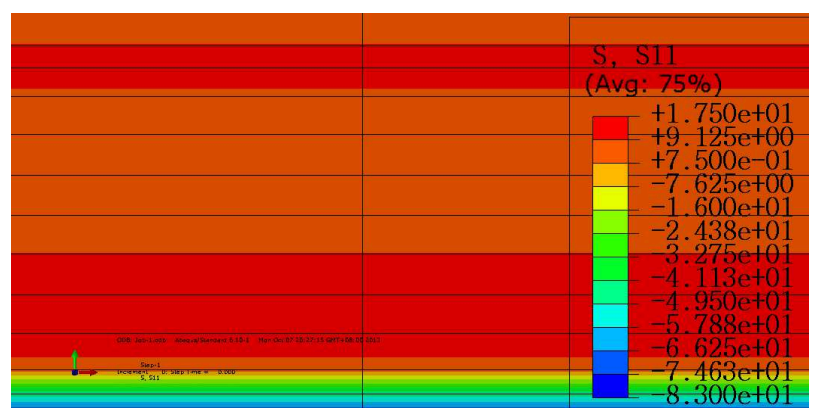

(a)Part of the model prior to the self-balanced state

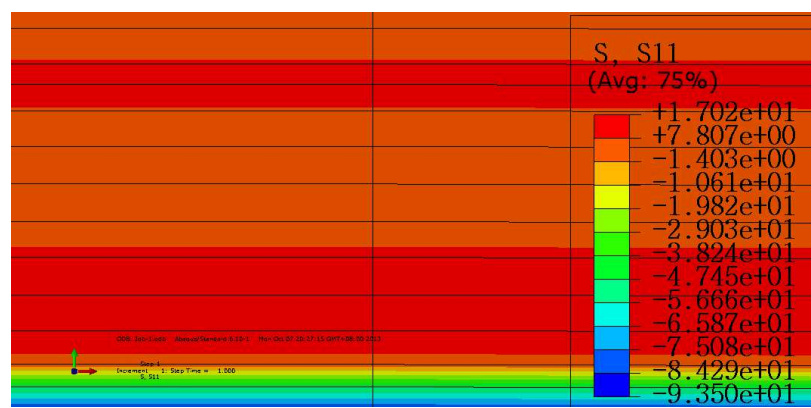

(b) Part of the model in the self-balanced state

Fig.7 The distribution of the stresses before and after the self-balanced state 


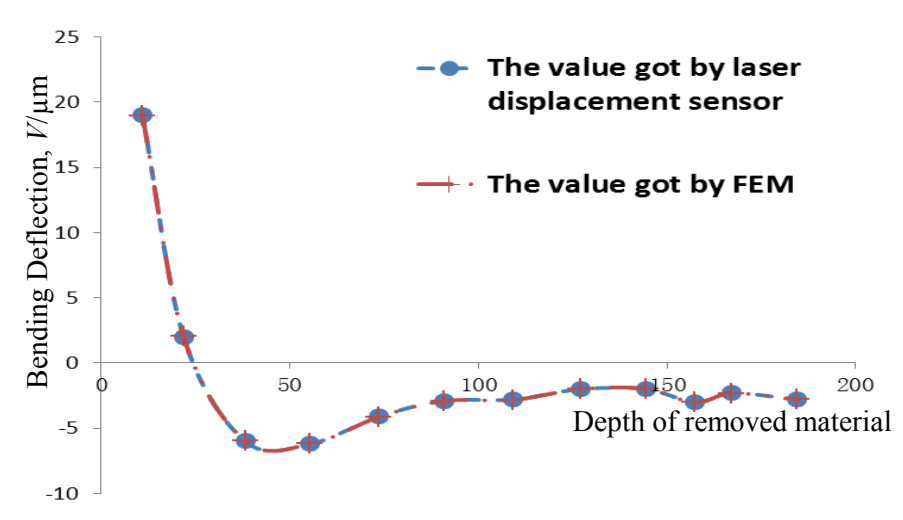

Fig. 8 The contrast of the bending deflection values got by measurement and that by FEM

\section{Summary}

The relationship between the changes of the bending deflection and the residual stresses has been analyzed, based on the deduced formulas and the measured bending deflections, the residual stresses can be calculated out. Applying the calculated stress values to the FEM model, and the analysis result has verified the correctness of this measuring method.

(1) Based on the actual meaning of the bending moment and the changes of the bending moment, the stress values were calculated out from the bottom layer to the top layer, until the residual stresses in all layers have been calculated out;

(2) Chemical milling was used to remove the material and release the residual stresses layer by layer, measuring the thickness of the removed layer and the change of the work piece's bending deflection provides reliable dates for calculating the residual stresses;

(3) Comparing the changes of the bending deflection calculated by FEM and that got in the experiments, it can be seen that they are coincident with each other very well. So it can be concluded that the stress values got by this method can predict deformations of workpieces with different sizes and shapes accurately, and it is very helpful to improve the accuracy of the workpieces in practice.

\section{References}

[1] X X Yu, W S Lau, T C Lee. Tools Manufact[J], 1997, 37 (10): 1525-1537

[2] C Liu, J X Zhang, B Wu, et al. Materials and Design[J], 2012, 34: 609-617

[3] C Xu. Manufacturing Technology \& Machine Tool[J], 2008(1): 80-82, 87

[4] A Mirzaee-Sisan, A J Fookes, C E Truman. International Journal of Pressure Vessels and Piping[J], 2007, 84: $265-273$

[5] X Ficquet, D J Smith, C E Truman. International Journal of Pressure Ve-ssels and Piping[J], 2009, 86: 20-30

[6] V G Navas, O Gonzalo, I Bengoetxea. International Journal of Machine Tools \& Manufacture[J], 2012, 61: 48-57

[7] S Pratihar, V Stelmukh, M T Hutchings.Materials Science and Engineering[J], 2006 A (437): $46-53$

[8] M Sebastiani, C Eberl, E Bemporad. Materials Science and Engineering[J], 2011, A(528): 7901-7908 
[9] S Marco, E Christoph, B Edoardo. Materials Science and Engineering[J], 2011,A(528): 7901-7908

[10] Y F Yang. Study on Prediction and Cotrol Technology of Machining Distortion for Monolithic Components Based on Inner Stress Field [D]. NanjingNanjing University of Aeronautics and Astronautics:2010

[11]M S Yin, et al. Material Protection [J], 2005, 38(8): 24-25, 41 